PROCEEDINGS OF THE

AMERICAN MATHEMATICAL SOCIETY

Volume 127, Number 5, Pages 1483-1491

S 0002-9939(99)05060-1

Article electronically published on January 29, 1999

\title{
COMPOSITION OPERATORS ON HARDY SPACES OF A HALF-PLANE
}

\author{
VALENTIN MATACHE
}

(Communicated by David R. Larson)

\begin{abstract}
We consider composition operators on Hardy spaces of a halfplane. We mainly study boundedness and compactness. We prove that on these spaces there are no compact composition operators.
\end{abstract}

\section{INTRODUCTION}

A lot of work has been done studying composition operators acting on Hardy spaces of functions holomorphic on the open unit disk $\mathbb{D}=\{z \in \mathbb{C}:|z|<1\}$. Composition operators on Hardy spaces of functions holomorphic on a half-plane enjoyed considerably less attention. This paper considers composition operators on the Hardy spaces $H^{p}(\mathbb{P}), 0<p<\infty$ where $\mathbb{P}=\{z \in \mathbb{C}: \operatorname{Im} z>0\}$ denotes the upper half-plane. Characterizing the compact composition operators acting on Hardy spaces of the open unit disk was a rather difficult job begun in a 1973 paper by J. H. Shapiro and J. H. Taylor and finished by J. H. Shapiro in a 1987 paper. The details concerning this subject can be found in [1] and [8]. The principal goal of this paper is to solve the same problem for Hardy spaces of a half-plane. The main result is Theorem 3.1 where we prove by contradiction that compact, composition operators acting on these spaces do not exist. The problem was first raised in the late seventies and early eighties in such papers as [9], [12], and [13] but remained unsolved until now. Section 2 in this paper is dedicated to setting up the notations and studying the boundedness of composition operators. Theorem 2.1 is an older result of this author [6], published in a little circulated journal. We decided to review it in this section in order to make this paper as self-contained as possible. Section 3 is dedicated to proving the main result.

\section{BOUNDEDNESS}

We begin this section by introducing some notations that we will use in the sequel. For each $f: \mathbb{P} \rightarrow \mathbb{C}$ holomorphic on $\mathbb{P},\|f\|_{p}$ is defined by

$$
\|f\|_{p}=\sup _{y>0}\left(\int_{\mathbb{R}}|f(x+i y)|^{p} d x\right)^{\frac{1}{p}} .
$$

Received by the editors September 1, 1997.

1991 Mathematics Subject Classification. Primary 47B38; Secondary 47B10.

Key words and phrases. Composition operator, Hardy space, compact operator.

(C)1999 American Mathematical Society 
Recall that $H^{p}(\mathbb{P})$ consists of all functions $f$, holomorphic on $\mathbb{P}$ for which $\|f\|_{p}$ is finite, that for $p \geq 1,\|\|_{p}$ is a Banach space norm (even a Hilbert space norm if $p=2$ ), and that for $0<p<1$

$$
d(f, g)=\|f-g\|_{p}^{p}, \quad f, g \in H^{p}(\mathbb{P}),
$$

defines a Fréchet space distance on $H^{p}(\mathbb{P})$. For any fixed selfmapping of $\mathbb{P}, \psi: \mathbb{P} \rightarrow$ $\mathbb{P}$ the composition operator induced by $\psi, C_{\psi}$ is the linear operator on the space of all $\mathbb{C}$-valued functions on $\mathbb{P}, \mathbb{C}^{\mathbb{P}}$ given by

$$
C_{\psi} f=f \circ \psi, \quad f \in \mathbb{C}^{\mathbb{P}} .
$$

We are interested in the situation when for some $p, 0<p<\infty, H^{p}(\mathbb{P})$ is left invariant by $C_{\psi}$, i.e., $C_{\psi} H^{p}(\mathbb{P}) \subseteq H^{p}(\mathbb{P})$. We wish to observe that, due to the closed graph principle and the well-known inequality

$$
|f(w)| \leq\left(\frac{2}{\pi \operatorname{Im} w}\right)^{\frac{1}{p}}\|f\|_{p}, \quad f \in H^{p}(\mathbb{P}), w \in \mathbb{P} ;
$$

this means that the restriction of $C_{\psi}$ to $H^{p}(\mathbb{P})$ is a bounded, linear operator on $H^{p}(\mathbb{P})$. This restriction operator will be equally denoted by $C_{\psi}$, and in such a situation we say that $\psi$ induces a bounded composition operator on $H^{p}(\mathbb{P})$. The conformal mapping $\gamma$ given by

$$
\gamma(z)=i \frac{1+z}{1-z}
$$

transforms $\mathbb{D}$ onto $\mathbb{P}$ and has inverse

$$
\gamma^{-1}(w)=\frac{w-i}{w+i} .
$$

Observe that if $d \theta$ is the arc-length Lebesgue measure on the unit circle $\mathbb{T}=\{z \in$ $\mathbb{C}:|z|=1\}$ and $d x$ is the Lebesgue measure on $\mathbb{R}$, then if we denote $\gamma\left(e^{i \theta}\right)=$ $i\left(1+e^{i \theta}\right) /\left(1-e^{i \theta}\right)=x$, we have $e^{i \theta}=(x-i) /(x+i)$ and hence

$$
d \theta=\frac{2}{1+x^{2}} d x .
$$

To each selfmapping $\psi$ of $\mathbb{P}$ we attach a selfmapping $\phi$ of $\mathbb{D}$ as follows:

$$
\phi=\gamma^{-1} \circ \psi \circ \gamma
$$

Suppose now that $\psi$ is analytic on $\mathbb{P}$. For each fixed $y>0$ the measurable mapping $\psi_{y}(x)=\psi(x+i y), x \in \mathbb{R}$ naturally induces a Borel measure on $\mathbb{P}, m \psi_{y}^{-1}$ called the pull-back measure induced by $\psi_{y}$

$$
m \psi_{y}^{-1}(E)=|\{x \in \mathbb{R}: \psi(x+i y) \in E\}|
$$

for each Borel subset $E \subseteq \mathbb{P}$. In (9) $|\quad|$ denotes the Lebesgue measure on $\mathbb{R}$. If $\psi$ induces a bounded composition operator on $H^{p}(\mathbb{P})$ for some $p, 0<p<\infty$, we can write

$$
\begin{gathered}
\int_{\mathbb{R}}|f(\psi(x+i y))|^{p} d x=\int_{\mathbb{R}}\left|\left(f \circ \psi_{y}\right)(x)\right|^{p} d x \\
=\int_{\mathbb{P}}|f|^{p} d m \psi_{y}^{-1} \leq\left\|\left.C_{\psi} f\right|_{p} ^{p} \leq\right\| C_{\psi}\left\|^{p}\right\| f \|_{p}^{p}, \quad f \in H^{p}(\mathbb{P}) .
\end{gathered}
$$

Above we made use of a well-known theorem in measure theory ([4], p. 163) in order to change the measure. Observe that (10) implies that the family $\left\{m \psi_{y}^{-1}\right\}_{y>0}$ is a 
family of Carleson measures on $\mathbb{P}$ with common bound, i.e., there exists $c>0$ such that

$$
m \psi_{y}^{-1}(\{w \in \mathbb{P}: t<\operatorname{Re} w<t+h, 0<\operatorname{Im} w<h\}) \leq c h
$$

for any $h>0, t \in \mathbb{R}$, and $y>0$ ([3], Theorem II3.9). The converse of this implication is also true because the theorem in [3] referred above gives necessary and sufficient conditions for a measure to be a Carleson measure, i.e., if (11) holds then (10) must hold with a constant $K$ instead of $\left\|C_{\psi}\right\|^{p}$; the same for all $y>0$. Therefore $C_{\psi} H^{p}(\mathbb{P}) \subseteq H^{p}(\mathbb{P})$. Summarizing these simple facts based on Carleson's theorem we obtain the following theorem which is the main result in [6].

Theorem 2.1. For any $p, 0<p<\infty$ an analytic map $\psi: \mathbb{P} \rightarrow \mathbb{P}$ induces a bounded composition operator on $H^{p}(\mathbb{P})$ if and only if the pull-back measures $m \psi_{y}^{-1}$, $y>0$ are Carleson measures with common bound $c$, i.e., if and only if there is a $c>0$ such that (11) holds for any $h>0, t \in \mathbb{R}$, and $y>0$.

The main advantage of this characterization of boundedness is that it proves that an analytic map $\psi: \mathbb{P} \rightarrow \mathbb{P}$ simultaneously induces bounded composition operators on all $H^{p}(\mathbb{P})$-spaces. We shall denote by $Q_{t, h}$ the open square $\{w \in \mathbb{P}: t<\operatorname{Re} w<$ $t+h, 0<\operatorname{Im} w<h\}$ which appears in (11). With this notation we prove the following.

Corollary 2.2. If $\psi$ is a bounded, analytic selfmapping of $\mathbb{P}$, then $\psi$ does not induce a bounded composition operator on any of the spaces $H^{p}(\mathbb{P}), 0<p<\infty$.

Proof. Choose $h>0$ big enough, such that $\psi(\mathbb{P}) \subseteq Q_{-h, 2 h}$, which is possible because $\psi$ is bounded. We have that for any $y>0, \psi_{y}^{-1}\left(Q_{-h, 2 h}\right)=\mathbb{R}$, so $m \psi_{y}^{-1}\left(Q_{-h, 2 h}\right)=\infty$, and therefore (11) does not hold.

Example 2.3. The only linear fractional mappings $\psi$ which leave $\mathbb{P}$ invariant and induce bounded composition operators on the $H^{p}(\mathbb{P})$-spaces are those of the form $\psi(w)=\alpha w+\beta$ with $\alpha>0$ and $\operatorname{Im} \beta \geq 0$.

Proof. We consider transforms of the form $\psi(w)=(a w+b) /(c w+d)$. By the previous corollary, we must have $c=0$, so $\psi$ must be of the form $\psi(w)=\alpha w+\beta$. Indeed, if $c \neq 0$, and the zero of the denominator has negative imaginary part, then $\psi$ is bounded on $\mathbb{P}$. If the denominator has its zero on the real line, say at $x$, observe one can choose $t \in \mathbb{R}$ such that $t<x<t+h$. In that case, there is a neighbourhood of $\infty$, i.e., a set $N$ of the form $\mathbb{C} \backslash D$ where $D$ is a closed disk such that $\psi(N) \subseteq Q_{t, h}$. Therefore for some $y>0$ big enough we shall have $\psi_{y}^{-1}\left(Q_{t, h}\right)=\mathbb{R}$, so $m \psi_{y}^{-1}\left(Q_{t, h}\right)=\infty$. We deduce $\psi$ must be of the form $\psi(w)=\alpha w+\beta$. $\alpha$ cannot be 0 because in that case $\psi$ would be bounded. Since $\psi$ must leave $\mathbb{P}$ invariant, we must have $\alpha>0$ and $\operatorname{Im} \beta \geq 0$. The fact that each such mapping satisfies condition (11) is immediate.

Consider the branch of $\sqrt{w}$ which maps $\mathbb{P}$ onto the first quadrant.

Example 2.4. $\psi(w)=\sqrt{w}$, does not induce a bounded composition operator on any of the $H^{p}(\mathbb{P})$-spaces.

Proof. Fix any $y_{0}>0$ and set $w=s+i y_{0} / 2 s$. So $w^{2}=s^{2}-y_{0}^{2} / 4 s^{2}+i y_{0}$. Therefore if $h=y_{0} / 2 t, \sqrt{w^{2}}=w \in Q_{t, h}$ for each $s, 0<t<s<t+h$. So 


$$
\begin{aligned}
& \left(s^{2}-y_{0}^{2} / 4 s^{2}\right) \in \psi_{y_{0}}^{-1}\left(Q_{t, h}\right) \text { if } 0<t<s<t+h \text { and hence } \\
& \begin{aligned}
\frac{\left|\left\{x \in \mathbb{R}: \sqrt{x+i y_{0}} \in Q_{t, h}\right\}\right|}{h} \geq \frac{(t+h)^{2}-y_{0}^{2} / 4(t+h)^{2}+y_{0}^{2} / 4 t^{2}-t^{2}}{h} \\
=h+2 t+\frac{y_{0}^{2}(h+2 t)}{4 t^{2}(t+h)^{2}} \rightarrow \infty
\end{aligned}
\end{aligned}
$$

if $t \rightarrow \infty$, so (11) cannot hold.

The selfmappings $\phi$ of the unit disk $\mathbb{D}$ induce bounded composition operators on all spaces $H^{p}(\mathbb{D})$ if and only if they are holomorphic on $\mathbb{D}$, their holomorphy being necessary because $f(z)=z, z \in \mathbb{D}$ is an $H^{p}(\mathbb{D})$-function for each $p, 0<p<\infty$, and $f \circ \phi=\phi$ must be in $H^{p}(\mathbb{D})$. The Hardy spaces on $\mathbb{P}$ have worse properties, so far as composition operators are concerned. As we saw, there exist analytic selfmappings of $\mathbb{P}$, even linear fractional transforms which leave $\mathbb{P}$ invariant, and do not induce bounded composition operators on any of the $H^{p}(\mathbb{P})$-spaces, $0<p<\infty$. It is also unclear if the fact that $\psi$ induces a bounded composition operator on $H^{p}(\mathbb{P})$, for some $p, 0<p<\infty$ implies that $\psi$ must be analytic on $\mathbb{P}$. In connection with that we make the following remark:

Remark. For any fixed $p, 0<p<\infty$, if a continuous selfmapping of $\mathbb{P}, \psi: \mathbb{P} \rightarrow \mathbb{P}$ induces a bounded composition operator on $H^{p}(\mathbb{P})$, then $\psi$ must be holomorphic on $\mathbb{P}$.

Proof. It will be enough to show that if $C_{\psi} H^{p}(\mathbb{P}) \subseteq H^{p}(\mathbb{P})$, then $\psi$ is analytic on $\mathbb{P}$. It is well-known that $f(w)=1 /(w+i)^{\frac{2}{p}}, w \in \mathbb{P}$ is an $H^{p}(\mathbb{P})$-function; hence $f \circ \psi \in H^{p}(\mathbb{P})$, so $f \circ \psi$ is analytic on $\mathbb{P}$. It is easy to see that $f^{\prime}(w) \neq 0, \forall w \in \mathbb{P}$, so $f$ is locally invertible with holomorphic local iverses, i.e., for each arbitrary $w_{0} \in \mathbb{P}$ we can choose an open neighbourhood $N$ of $f\left(\psi\left(w_{0}\right)\right)$ where $f$ has a holomorphic inverse $f^{-1}$, and an open neighbourhood $V$ of $w_{0}$, such that $(f \circ \psi)(V) \subseteq N$ so, for each $w \in V$ we have $f^{-1} \circ f \circ \psi(w)=\psi(w)$, which shows that $\psi$ must be holomorphic on $V$. Since $w_{0}$ was arbitrarily chosen we deduce that $\psi$ is analytic on $\mathbb{P}$.

In the sequel we shall always consider holomorphic selfmappings of $\mathbb{P}$. For any $f \in H^{p}(\mathbb{P}) f(x)=\lim _{y \rightarrow 0_{+}} f(x+i y)$ is known to exist a.e. on $\mathbb{R}$, and will be referred to as the boundary function of $f$. In connection with that we prove

Proposition 2.5. If $\psi: \mathbb{P} \rightarrow \mathbb{P}$ is holomorphic, the boundary function of $\psi, \psi(x)=$ $\lim _{y \rightarrow 0_{+}} \psi(x+i y)$ exists and is finite for almost every $x \in \mathbb{R}$.

Proof. Suppose $\phi$ is the function in (8) and $\phi\left(e^{i \theta}\right)$ is its boundary function which exists a.e. on the unit circle. Since $\gamma^{-1}$ transforms the vertical line through $x$ into a circle passing through 1 and $e^{i \theta}=(x-i) /(x+i)$, for each $x \in \mathbb{R}$, the boundary function $\psi(x)$ exists for each $e^{i \theta}=(x-i) /(x+i)$ for which $\phi\left(e^{i \theta}\right)$ exists. Therefore, if $E \subseteq \mathbb{R}$ is the set of all $x \in \mathbb{R}$ where $\psi(x)$ does not exist, then the set $\gamma^{-1}(E) \subseteq \mathbb{T}$ must have measure 0 . Hence we can write

$$
\int_{0}^{2 \pi} \chi_{\gamma^{-1}(E)}\left(e^{i \theta}\right) \frac{d \theta}{2 \pi}=\int_{0}^{2 \pi} \chi_{E} \circ \gamma\left(e^{i \theta}\right) \frac{d \theta}{2 \pi}=0
$$

which, by (7) implies $1 / \pi \int_{\mathbb{R}} \chi_{E}(x) /\left(1+x^{2}\right) d x=0$. This means $\chi_{E}=0$ a.e., i.e., $\psi(x)$ exists a.e. on $\mathbb{R}$. If for some $x \in \mathbb{R}, \psi(x)=\infty$, then $\phi\left(e^{i \theta}\right)=1$. So if $E$ denotes 
the set of all $x \in \mathbb{R}$ for which this happens, then $\gamma^{-1}(E) \subseteq\left\{e^{i \theta} \in \mathbb{T}: \phi\left(e^{i \theta}\right)=1\right\}$; hence $\gamma^{-1}(E)$ must have measure 0 because $\phi$ is not constantly equal to 1 , since it is a selfmapping of $\mathbb{D}$. By the same computation as above we obtain that $E$ must have measure 0 , i.e., $\psi(x)$ is finite for almost all $x \in \mathbb{R}$.

For each mapping $\varphi: \mathbb{D} \rightarrow \mathbb{C}$, the multiplication operator induced by $\varphi, M_{\varphi}$ is the linear mapping

$$
M_{\varphi} f=\varphi f, \quad f \in \mathbb{C}^{\mathbb{D}},
$$

where $\mathbb{C}^{\mathbb{D}}$ is the linear space of all $\mathbb{C}$-valued mappings on $\mathbb{D}$. For each selfmapping $\psi$ of $\mathbb{P}$, attach $\phi$ to $\psi$ as in (8), and denote by $\omega_{p}$ the function

$$
\omega_{p}(z)=\frac{(1-\phi(z))^{\frac{2}{p}}}{(1-z)^{\frac{2}{p}}}, \quad z \in \mathbb{D} .
$$

We denote by $A_{p}$ the linear mapping

$$
A_{p} f=M_{\omega_{p}} C_{\phi} f, \quad f \in \mathbb{C}^{\mathbb{D}} .
$$

For each $p, 0<p<\infty$ we make the notation

$$
\varphi_{p}(z)=(1-z)^{\frac{2}{p}}, \quad z \in \mathbb{D},
$$

a simple computation shows that

$$
M_{\varphi_{p}} A_{p} f=C_{\phi} M_{\varphi_{p}} f, \quad f \in \mathbb{C}^{\mathbb{D}} .
$$

With this notation we can prove the following theorem which extends a result in [11].

Theorem 2.6. If $\psi$ is a selfmapping of $\mathbb{P}$ and $p$ is fixed, $0<p<\infty$, the following are equvalent:

$$
\begin{aligned}
C_{\psi} H^{p}(\mathbb{P}) & \subseteq H^{p}(\mathbb{P}), \\
A_{p} H^{p}(\mathbb{D}) & \subseteq H^{p}(\mathbb{D}), \\
C_{\phi} M_{\varphi_{p}} H^{p}(\mathbb{D}) & \subseteq M_{\varphi_{p}} H^{p}(\mathbb{D}), \\
A_{2} H^{2}(\mathbb{D}) & \subseteq H^{2}(\mathbb{D}) .
\end{aligned}
$$

Proof. The equivalence $(17) \Leftrightarrow(18)$ is proven in [11] for $p=2$. The same proof works for any $p$, i.e., the linear transforms

$$
V f(w)=\frac{1}{\pi^{\frac{1}{p}}(w+i)^{\frac{2}{p}}} f\left(\frac{w-i}{w+i}\right), \quad w \in \mathbb{P}, f \in \mathbb{C}^{\mathbb{D}},
$$

and

$$
W g(z)=\frac{e^{\frac{i \pi}{p}}(4 \pi)^{\frac{1}{p}} g\left(i \frac{1+z}{1-z}\right)}{(1-z)^{\frac{2}{p}}}, \quad z \in \mathbb{D}, g \in \mathbb{C}^{\mathbb{P}},
$$

are inverse to each other. Checking this is a routine computation. One can use (7) to check that the restrictions of $V$ and $W$ to $H^{p}(\mathbb{D})$ and $H^{p}(\mathbb{P})$ respectively, are isometric, so regarded as mappings between $H^{p}(\mathbb{D})$ and $H^{p}(\mathbb{P}) V$ and $W$ are onto isometries, inverse to each other. It is routine to check that $W C_{\psi} V f=A_{p} f$, for any $f \in H^{p}(\mathbb{D})$ so $\psi$ induces a bounded composition operator on $H^{p}(\mathbb{P})$ if and only if $A_{p}$ restricted to $H^{p}(\mathbb{D})$ is a bounded operator on $H^{p}(\mathbb{D})$ which, by the 
closed graph principle happens if and only if $A_{p} H^{p}(\mathbb{D}) \subseteq H^{p}(\mathbb{D})$, i.e., $(17) \Leftrightarrow(18)$. Since an analytic selfmapping of $\mathbb{P}$ simultaneously induces bounded composition operators on all $H^{p}(\mathbb{P})$-spaces, we deduce $(17) \Leftrightarrow(20)$. If (18) holds, then by (16) $C_{\phi} M_{\varphi_{p}} H^{p}(\mathbb{D})=M_{\varphi_{p}} A_{p} H^{p}(\mathbb{D}) \subseteq M_{\varphi_{p}} H^{p}(\mathbb{D})$, i.e., (18) $\Rightarrow(19)$. If (19) holds, this means that for each $f \in H^{p}(\mathbb{D})$ there is some $g \in H^{p}(\mathbb{D})$ such that $C_{\phi}\left(\varphi_{p} f\right)=\varphi_{p} g$, i.e., $(1-\phi(z))^{2 / p}(f \circ \phi)(z)=(1-z)^{2 / p} g(z), z \in \mathbb{D}$, i.e., such that $A_{p} f=g$, which means that $A_{p} H^{p}(\mathbb{D}) \subseteq H^{p}(\mathbb{D})$, i.e., $(19) \Rightarrow(17)$.

Clearly, since a selfmapping of $\mathbb{P}$ simultaneously induces bounded composition operators on all $H^{p}(\mathbb{P})$-spaces, the number 2 in $(20)$ can be replaced by any $q$, $0<q<\infty$. However, since $H^{2}(\mathbb{D})$ is a Hilbert space it is more comfortable for us to work with 2 rather than a different $q$.

Corollary 2.7. If $\psi: \mathbb{P} \rightarrow \mathbb{P}$ induces a bounded composition operator on $H^{p}(\mathbb{P})$, for some $p, 0<p<\infty$, then $\omega_{2} \in H^{2}(\mathbb{D})$. If $\omega_{2}$ is bounded, then $\psi$ induces a bounded composition operator on each $H^{p}(\mathbb{P})$-space.

Proof. If $C_{\psi}$ is bounded on $H^{p}(\mathbb{P})$, observe that the constant function 1 is an $H^{2}(\mathbb{D})$-function and $A_{2} 1=\omega_{2}$ so $\omega_{2} \in H^{2}(\mathbb{D})$. If $\omega_{2}$ is bounded, then both $M_{\omega_{2}}$ and $C_{\phi}$ are bounded operators on $H^{2}(\mathbb{D})$, therefore so is $A_{2}$.

In Example 2.4, $\psi(w)=\sqrt{w}$ was shown not to induce a bounded composition operator. Observe that, in that case $\psi^{\prime}$ is not bounded below. This is significant as we can see from the following

Theorem 2.8. If $\psi: \mathbb{P} \rightarrow \mathbb{P}$ is analytic on $\mathbb{P}$, and the function $\eta(w, z)=\mid \operatorname{Re} \psi^{\prime}(w)$ $+i \operatorname{Im} \psi^{\prime}(z) \mid, z, w \in \mathbb{P}$ is bounded below, then $\psi$ induces a bounded composition operator on the $H^{p}(\mathbb{P})$-spaces.

Proof. Choose $\epsilon>0$ such that $|\eta(w, z)| \geq \epsilon$ for all $z, w \in \mathbb{P}$. For $\psi=u+i v$, this means $|\partial u(w) / \partial x+i \partial v(z) / \partial x| \geq \epsilon, \forall z, w \in \mathbb{P}$. Take $t \in \mathbb{R}, y>0, h>0$ and observe that if $\psi\left(x_{1}+i y\right), \psi\left(x_{2}+i y\right) \in Q_{t, h}$, then $\left|\psi\left(x_{1}+i y\right)-\psi\left(x_{2}+i y\right)\right| \leq \sqrt{2} h$. On the other hand, by the mean value theorem, there exist $s_{1}, s_{2}$ between $x_{1}$ and $x_{2}$ such that

$$
\left|\psi\left(x_{1}+i y\right)-\psi\left(x_{2}+i y\right)\right|=\left|\frac{\partial u\left(s_{1}+i y\right)}{\partial x}+i \frac{\partial v\left(s_{2}+i y\right)}{\partial x}\right|\left|x_{1}-x_{2}\right|
$$

so $\left|x_{1}-x_{2}\right| \leq \sqrt{2} h / \epsilon ;$ hence

$$
m \psi_{y}^{-1}\left(Q_{t, h}\right) \leq \sup \left\{\left|x_{1}-x_{2}\right|: x_{1}, x_{2} \in \psi_{y}^{-1}\left(Q_{t, h}\right)\right\} \leq \frac{\sqrt{2} h}{\epsilon}
$$

which by Theorem 2.1 proves that $C_{\psi}$ is bounded.

Example 2.9. For any $\alpha>0$ and $\beta \in \mathbb{C}, \operatorname{Im} \beta \geq 0$ the function $\psi(w)=\alpha w+\beta+$ $\sqrt{w}, w \in \mathbb{P}$ induces a bounded composition operator on the $H^{p}(\mathbb{P})$-spaces.

Proof. Since $\mathbb{P}$ is an additive semigroup and both $\alpha w+\beta$ and $\sqrt{w}, w \in \mathbb{P}$ are selfmappings of $\mathbb{P}$, we deduce $\psi(\mathbb{P}) \subseteq \mathbb{P}$. Observe that $\psi^{\prime}(w)=\alpha+1 / 2 \sqrt{w}$ so, since $1 / 2 \sqrt{w}, w \in \mathbb{P}$ transforms $\mathbb{P}$ onto the 4 -th quadrant, and addition of $\alpha$ shifts the 4th quadrant to the right, we deduce that $\left|\operatorname{Re} \psi^{\prime}(w)\right| \geq \alpha>0$ for any $w \in \mathbb{P}$. Observe that $|\eta(w, z)| \geq\left|\operatorname{Re} \psi^{\prime}(w)\right| \geq \alpha>0, \forall w, z \in \mathbb{P}$, which means that $\psi$ satisfies the hypothesis of Theorem 2.8; hence $C_{\psi}$ is bounded. 
The good thing about condition (20) in Theorem 2.6 is that it reduces the problem of deciding if $\psi$ induces a bounded composition operator on the $H^{p}(\mathbb{P})$-spaces to checking if the weighted composition operator $A_{2}$ leaves $H^{2}(\mathbb{D})$ invariant. In connection with the mapping $\phi$ which appears in the definition of $A_{2}$, the authors of [10] observe that $\phi$ has angular derivative at 1 if $\omega_{2}$ is bounded which is a particular situation when $\psi$ induces a bounded composition operator (by Corollary 2.7). The following is a generalization of this statement.

Theorem 2.10. If $\psi: \mathbb{P} \rightarrow \mathbb{P}$ induces a bounded composition operator on the $H^{p}(\mathbb{P})$-spaces, then $\phi$ has angular derivative at 1 , and the angular limit of $\phi$ at 1 equals 1 .

Proof. According to Theorem 2.6, $\psi$ must induce a bounded composition operator on $H^{2}(\mathbb{P})$ and in that case, the quantity $\delta=\sup \{\operatorname{Im} w / \operatorname{Im} \psi(w): w \in \mathbb{P}\}$ must be finite. Indeed, this is a simple fact, first observed by the authors of [12]. By the Cauchy formula for $H^{2}(\mathbb{P})$-functions ([2], Theorem 11.8), the reproducing kernel of the reproducing kernel Hilbert space $H^{2}(\mathbb{P})$ consists of the functions $k_{\alpha}(w)=$ $i / 2 \pi(w-\bar{\alpha}), w \in \mathbb{P}$. By the reproducing property, the norm of such a function is $\left\|k_{\alpha}\right\|_{2}^{2}=k_{\alpha}(\alpha)=1 / \sqrt{4 \pi \operatorname{Im} \alpha}$. If we consider now the normalized reproducing kernel functions $g_{\alpha}=k_{\alpha} /\left\|k_{\alpha}\right\|_{2}$, use the well-known relation $C_{\psi}^{*} k_{\alpha}=k_{\psi(\alpha)}$ ([1], Theorem 1.4) and write $\left\|C_{\psi}^{*} g_{\alpha}\right\|_{2} \leq\left\|C_{\psi}^{*}\right\|, \forall \alpha \in \mathbb{P}$, we get $\delta \leq\left\|C_{\psi}^{*}\right\|^{2}<\infty$. This implies

$$
\psi(\mathbb{P}+i \lambda) \subseteq \mathbb{P}+i \lambda / \delta \quad \forall \lambda>0 .
$$

The Julia-Carathéodory theorem ([8], p. 57, or [1], Theorem 2.44) gives necessary and sufficient conditions for the existence of the angular derivative. Relation (23) is condition ( $\left.\mathrm{JC} 1 \frac{1}{2}\right)$ in the proof given for this theorem in [8], p. 69, transported in the upper half-plane instead of the right half-plane, and is equivalent to the existence of the $\operatorname{limit}_{\lim _{z \rightarrow 1}}(1-\phi(z)) /(1-z)$ as $z$ tends to 1 inside a nontangential approach region with vertex at 1 . Indeed, if $\mathbb{H}$ is the right, open half-plane and we define $\Psi(s)=\bar{i} \psi(i s), s \in \mathbb{H}$, then $\Psi=\tau^{-1} \circ \phi \circ \tau$, where $\tau(s)=(s-1) /(s+1), s \in \mathbb{H}$, and (23) is equivalent to

$$
\Psi(\mathbb{H}+\lambda) \subseteq \mathbb{H}+\lambda / \delta \quad \forall \lambda>0
$$

which is condition $\left(\mathrm{JC} 1 \frac{1}{2}\right)$. The existence of this angular derivative, obviously implies that the angular limit of $\phi$ at 1 must exist and be equal to 1 . This last fact is also proved separately in [10] by a different method, but in full generality, i.e., for all $\psi$ which induce bounded composition operators on $H^{2}(\mathbb{P})$.

\section{Compactness}

In this section we prove there are no compact composition operators on any of the spaces $H^{p}(\mathbb{P}), 1 \leq p<\infty$. The proof is by contradiction.

Theorem 3.1. If $\psi: \mathbb{P} \rightarrow \mathbb{P}$ induces a bounded composition operator $C_{\psi}$ on $H^{p}(\mathbb{P})$, $1 \leq p<\infty$, then $C_{\psi}$ is never compact.

Proof. Suppose first that $1<p<\infty$. By the Cauchy formula ([2], Theorem 11.8), the function in $\bar{H}^{q}(\mathbb{P}), q=(1-1 / p)^{-1}$, corresponding to the point evaluation functional

$$
\hat{\alpha} f=f(\alpha), \quad f \in H^{p}(\mathbb{P}), \alpha \in \mathbb{P},
$$


under the usual identification of the dual of $H^{p}(\mathbb{P})$ with $\bar{H}^{q}(\mathbb{P})$ ([3], Theorem VI, $4.2)$ is $k_{\alpha}=\overline{i / 2 \pi(w-\bar{\alpha})}, \alpha \in \mathbb{P}$. This means that there is a bounded, invertible operator $T:\left(H^{p}(\mathbb{P})\right)^{*} \rightarrow \bar{H}^{q}(\mathbb{P})$ such that $T \hat{\alpha}=k_{\alpha}, \alpha \in \mathbb{P}$. Let us observe that

$$
\begin{aligned}
\left\|k_{\alpha}\right\|_{q} & =\frac{1}{2 \pi}\left(\int_{\mathbb{R}} \frac{d t}{|t-\bar{\alpha}|^{q}}\right)^{\frac{1}{q}}=\frac{1}{2 \pi(\operatorname{Im} \alpha)^{\frac{q-1}{q}}}\left(\int_{\mathbb{R}} \frac{d t}{\left(1+t^{2}\right)^{\frac{q}{2}}}\right)^{\frac{1}{q}} \\
= & \frac{c}{(\operatorname{Im} \alpha)^{\frac{1}{p}}} \quad \text { where } \quad c=\frac{1}{2 \pi}\left(\int_{\mathbb{R}} \frac{d t}{\left(1+t^{2}\right)^{\frac{q}{2}}}\right)^{\frac{1}{q}} .
\end{aligned}
$$

Now set $\alpha_{n}=i n, n \geq 1$. Observe that if we define $g_{\alpha_{n}}=k_{\alpha_{n}} /\left\|k_{\alpha_{n}}\right\|_{q}$, then this is a norm-bounded sequence which converges to 0 pointwise on $\mathbb{P}$. Indeed, for each $w \in \mathbb{P}$ we have $g_{\alpha_{n}}(w)=\left(n^{\frac{1}{p}} / c\right)(\overline{i / 2 \pi(w+i n)})$. Since $1 / p<1$, it is obvious that $\left(g_{\alpha_{n}}\right)$ tends to 0 pointwise. For $1<p<\infty, H^{p}(\mathbb{P})$ is a reflexive space so, Corollary 1.3 in [1] works in these spaces; hence $g_{\alpha_{n}} \rightarrow 0$ weakly. If $C_{\psi}$ is compact, then $C_{\psi}^{*}$ is compact, so $T C_{\psi}^{*} T^{-1}$ is compact and, by a well-known property of composition operators ([1], Theorem 1.4) $T C_{\psi}^{*} T^{-1} g_{\alpha_{n}}=k_{\psi\left(\alpha_{n}\right)} /\left\|k_{\alpha_{n}}\right\|_{q}$. This sequence must tend to 0 in the norm, i.e., $\operatorname{Im} \alpha_{n} / \operatorname{Im} \psi\left(\alpha_{n}\right) \rightarrow 0$. We wish to show that this generates a contradiction, and deduce that $C_{\psi}$ cannot be compact. Denote $r_{n}=(n-1) /(n+1)$. We have $\alpha_{n}=i\left(1+r_{n}\right) /\left(1-r_{n}\right)$, i.e., $\alpha_{n}=\gamma\left(r_{n}\right)$. So $\operatorname{Im} \alpha_{n}=\left(\left(1+r_{n}\right) /\left(1-r_{n}\right)\right)=\left(1-r_{n}^{2}\right) /\left(1-r_{n}\right)^{2}$ and

$$
\operatorname{Im} \psi\left(\alpha_{n}\right)=\operatorname{Re} \frac{1+\phi\left(r_{n}\right)}{1-\phi\left(r_{n}\right)}=\frac{1-\left|\phi\left(r_{n}\right)\right|^{2}}{\left|1-\phi\left(r_{n}\right)\right|^{2}} .
$$

We deduce

$$
\frac{1-r_{n}^{2}}{\left(1-r_{n}\right)^{2}} / \frac{1-\left|\phi\left(r_{n}\right)\right|^{2}}{\left|1-\phi\left(r_{n}\right)\right|^{2}} \rightarrow 0
$$

that is,

$$
\lim _{n \rightarrow \infty} \frac{\left|1-\phi\left(r_{n}\right)\right|^{2}}{\left|1-r_{n}\right|^{2}} \frac{1-r_{n}^{2}}{1-\left|\phi\left(r_{n}\right)\right|^{2}}=0 .
$$

Denote by $\phi^{\prime}(1)$ the angular derivative of $\phi$ at 1 , which must exist (by Theorem 2.10) and be a nonzero number (see [1], the remark following Corollary 2.40 and Theorem 2.44 or [8], p. 57). We obtain that

$$
\left|\phi^{\prime}(1)\right|^{2} \lim _{n \rightarrow \infty} \frac{1-r_{n}^{2}}{1-\left|\phi\left(r_{n}\right)\right|^{2}}=0
$$

i.e., $\lim _{n \rightarrow 0}\left(1-r_{n}^{2}\right) /\left(1-\left|\phi\left(r_{n}\right)\right|^{2}\right)=0$. Since $\left(1-r_{n}^{2}\right) /\left(1-\left|\phi\left(r_{n}\right)\right|^{2}\right) \geq\left(1-r_{n}\right) /$ $2\left(1-\left|\phi\left(r_{n}\right)\right|\right)$, we deduce

$$
\lim _{n \rightarrow \infty} \frac{1-\left|\phi\left(r_{n}\right)\right|}{1-r_{n}}=\infty
$$

which is a contradiction because the limit above should be equal to $\left|\phi^{\prime}(1)\right|$ ([1], Theorem 2.44). This proves the result for $1<p<\infty$. For $p=1$, we now start by choosing a sequence $\left(g_{n}\right)_{n}$ in the closed unit ball of $H^{2}(\mathbb{P})$ which converges pointwise to 0 and such that $\left\|C_{\psi} g_{n}\right\|_{2} \geq \epsilon>0, \forall n \geq 1$. Such a sequence exists because we have just proved that $C_{\psi}$ is not a compact operator on $H^{2}(\mathbb{P})$. Now set $f_{n}=g_{n}^{2}$, $\forall n \geq 1$. We obtain a sequence in the unit ball of $H^{1}(\mathbb{P})$, since $\left\|f_{n}\right\|_{1}=\left\|g_{n}\right\|_{2}^{2}$, $\forall n \geq 1$. Suppose now that $C_{\psi}$ is a compact operator on $H^{1}(\mathbb{P})$. In that case, $\left(C_{\psi} f_{n}\right)_{n}$ must contain a norm convergent subsequence $\left(C_{\psi} f_{n_{k}}\right)_{k}$. Norm convergence 
in $H^{1}(\mathbb{P})$ implies pointwise convergence (by $(4)$ ), and $C_{\psi} f_{n_{k}}(w)=g_{n_{k}}^{2}(\psi(w)) \rightarrow 0$, $\forall w \in \mathbb{P}$. Therefore $\left(C_{\psi} f_{n_{k}}\right)_{k}$ must tend to 0 in the norm, which is a contradiction because $\left\|C_{\psi} f_{n_{k}}\right\|_{1}=\left\|C_{\psi} g_{n_{k}}\right\|_{2}^{2} \geq \epsilon^{2}>0, \forall k \geq 1$.

We finish this paper by raising the following

Problem. Give a nice characterization in terms of the symbol $\phi$ of those composition operators $C_{\phi}$ on $H^{2}(\mathbb{D})$ which leave invariant the operator range $(1-z) H^{2}(\mathbb{D})$.

Some comments are in order here. Observe that for $p=2$, the mapping defined in $(15)$ is $\varphi_{2}(z)=1-z, z \in \mathbb{D}$. The operator range above is the range of $I-S$ where $S$ is the unilateral forward shift of multiplicity 1 , i.e., the multiplication operator with the coordinate function acting on $H^{2}(\mathbb{D})$. By condition (19) in Theorem 2.6, solving this problem means characterizing those selfmappings $\phi$ of $\mathbb{D}$ associated by condition (8) to the selfmappings $\psi$ of $\mathbb{P}$ which induce bounded composition operators on the spaces $H^{p}(\mathbb{P})$. By the term "nice characterization" in the statement of this problem we mean a necessary and sufficient condition easier to work with than the necessary and sufficient conditions contained by Theorems 2.1 and 2.6. Observe that, by Theorem 2.10 such a $\phi$ must have angular derivative at 1 and the angular limit at 1 should be equal to 1 (i.e., 1 should be a boundary fixed point for $\phi$ ). By Corollary 2.7 another necessary condition $\phi$ needs to satisfy is that $\omega_{2}(z)=(1-\phi(z)) /(1-z)$, $z \in \mathbb{D}$ be an $H^{2}(\mathbb{D})$-function.

\section{REFERENCES}

1. C. C. Cowen and B. D. MacCluer, Composition Operators on Spaces of Analytic Functions, CRC Press Boca Raton, New York, London, Tokyo, 1995. MR 97i:47056

2. P. L. Duren, Theory of $H^{p}$ Spaces, Academic Press, New York, 1970. MR 42:3552

3. J. B. Garnett, Bounded Analytic Functions, Academic Press, New York, 1981. MR 83g:30037

4. P. R. Halmos, Measure Theory, Van Nostrand, Princeton, NJ, 1950. MR 11:504d

5. K. Hoffman, Banach Spaces of Analytic Functions, Englewood Cliffs, NJ, 1962. MR 24:A2844

6. V. Matache, Composition Operators on $H^{p}$ of the Upper Half-Plane, An. Univ. Timisoara Ser. Stiint. Mat. 27 (1989), 63-66. MR 92k:47063

7. E. A. Nordgren, Composition Operators in Hilbert Spaces, Hilbert Space Operators, Lecture Notes in Mathematics, vol. 693, Springer-Verlag, New York, Heidelberg, Berlin, 1978, pp. 3763. MR 80d: 47046

8. J. H. Shapiro, Composition Operators and Classical Function Theory, Springer-Verlag, New York, Heidelberg, Berlin, 1993. MR 94k:47049

9. S. D. Sharma, Compact and Hilbert-Schmidt Composition Operators on Hardy Spaces of the Upper Half-Plane, Acta Sci. Math. (Széged) 46 (1983), 197-202. MR 85g:47032

10. S. D. Sharma and R. K. Singh, Composition Operators on Hardy Spaces of the Upper HalfPlane, preprint (1996).

11. R. K. Singh, A Relation Between Composition Operators on $H^{2}(\mathbb{D})$ and $H^{2}\left(\Pi^{+}\right)$, Pure Appl. Math. Sci. 1 (1974), 1-5. MR 56:12971

12. R. K. Singh and S. D. Sharma, Composition Operators on a Functional Hilbert Space, Bull. Austral. Math. Soc. 20 (1979), 377-384. MR 81c:47034

13. R. K. Singh and S. D. Sharma, Noncompact Composition Operators, Bull. Austral. Math. Soc. 21 (1980), 125-130. MR 81m:47046

Department of Mathematics, University of Kansas, Lawrence, Kansas 66045-2142

E-mail address: matache@math.ukans.edu

Current address: Department of Mathematics, University of Puerto Rico, P. O. Box 9018, Mayagüez, Puerto Rico 00681-9018

E-mail address: matache@math.upr.clu.edu 